\title{
INFORMATION
}

\section{SOCIÉté DES ACARologues DE LANGUE FRANÇAISE (S.A.L.F.) : Cours européen d'Acarologie.}

17-30 septembre 1984. Station biologidue de Paimpont (université de Rennes).

Séminaire d'Acarologie fondamentale et appliquée de haut niveau destiné à un public d'étudiants, de chercheurs, de praticiens, de techniciens et d'ingénieurs. Cycle de deux de semaines conférences et de travaux pratiques; acarologie générale en première semaine et ateliers spécialisés en deuxième semaines :

- Acarologie fondamentale,

- Acariens et tiques d'importance médicale et vétérinaire,

- Acariens des denrées conservées,

- Acariens phytoparasites et méthodes de lutte,

- Acariens du sol.

Renseignements et inscriptions : Dr F. Athias-Binche. Secrétaire Général de la S.A.L.F. — Laboratoire Arago 66650. 6650 Banyuls-sur-mer, France. 